\title{
Experimental Organism Malignant Oligodendroglioma
}

National Cancer Institute

\section{Source}

National Cancer Institute. Experimental Organism Malignant Oligodendroglioma. NCI

Thesaurus. Code C119577.

A malignant neoplasm of the central nervous system arising from oligodendrocytes. 